\title{
Providing a teaching and learning open and innovative toolkit for evidence-based practice to nursing European curriculum (EBP e-Toolkit): Project rationale and design
}

\author{
Evridiki Patelarou ${ }^{1,2}$, Konstantinos Vlasiadis ${ }^{3}$, Mariusz Panczyk ${ }^{1,4}$, Jakub Dolezel $^{1,5}$, Darja Jarosova ${ }^{1,5}$, Joanna Gotlib ${ }^{1,4}$, Brigita \\ Skela-Savič ${ }^{1,6}$, Daniela Mecugni ${ }^{1,7}$, Stefano Finotto ${ }^{1,7}$, Maria Ruzafa-Martinez ${ }^{1,8}$, Antonio Jesús Ramos-Morcillo ${ }^{1,2}$, Athina \\ Patelarou ${ }^{1,2}$
}

\begin{abstract}
AFFILIATION
1 European Nursing Centre for Learning Evidence-Based Practice (ENCLEBP), Murcia, Spain

2 Department of Nursing, Faculty of Health Sciences, Hellenic Mediterranean University, Heraklion, Greece

3 Dental Clinic, General University Hospital of Heraklion, Heraklion, Greece 4. Department of Education and Research in Health Sciences, Faculty of Health Sciences, Medical University of Warsaw, Poland

5 Department of Nursing and Midwifery, Faculty of Medicine, University of Ostrava, Ostrava, Czech Republic

6 Angela Boškin Institute for Research in Healthcare Sciences, Angela Boškin Faculty of Health Care, Jesenice, Slovenia

7 Department of Surgery, Medicine, Dentistry and Morphological
\end{abstract}

Sciences, University of Modena and Reggio Emilia, Degree Course in Nursing, seat of Reggio Emilia, Reggio Emilia, Italy 8 College of Nursing, University of Murcia, Murcia, Spain

\section{CORRESPONDENCE TO}

Evridiki Patelarou. Department of Nursing, Faculty of Health Sciences, Hellenic Mediterranean University, 71414 Crete, Greece. E-mail: epatelarou@hmu.gr

\section{KEYWORDS}

evidence-based practice, competence, curriculum, nursing

Received: 4 August 2020, Accepted: 8 October 2020

\begin{abstract}
Evidence-based practice (EBP) is an instrument of great utility in making clinical decisions in nursing care, improving the quality of nursing care and patients' health outcomes. There is no European framework for EBP competency, and no guidelines for EBP teaching. The general concept of the project 'Providing a Teaching and Learning Open and Innovative Toolkit for Evidence-based Practice to Nursing European Curriculum: (EBP e-Toolkit)' is to fill this gap in Nursing education across Europe and to foster and harmonize the teaching and learning of EBP in the European nursing
\end{abstract}

curricula and to produce the acquisition of EBP competence earlier in professional life. The project is organized along four major outputs, and a dissemination and sustainability plan has been set up. A mixed method research constitutes the main methodological approach applied in the project. This methodology requires the active participation of all research groups, partners, steering committee, nursing students, and educators, in the project. The use of the EBP e-Toolkit will increase nurses' and nursing students' level of EBP competence by formulating specific guidelines to be implemented in EBP teaching.

\section{INTRODUCTION}

Evidence-based practice (EBP) is a problem-solving approach to clinical decision-making that integrates the best available scientific evidence with clinical experience and patients' preferences and values ${ }^{1}$. EBP is an instrument of great utility in making clinical decisions in nursing care, helping to reduce healthcare $\operatorname{costs}^{2}$ and variability in clinical practice, improving the quality of nursing care practice and patients' health outcomes ${ }^{3}$, and increasing job satisfaction of nursing professionals ${ }^{4}$.

EBP has been declared as a professional responsibility and central characteristic of the work of nurses by the International Council of Nurses, which highlights that nurse staffing decisions must be evidence-based ${ }^{5}$. In addition, the European Federation of Nurses Associations developed a Competency Framework ( $\mathrm{CF}$ ), based in the European Directive 2013/55/EU, that establishes the minimum educational requirements for nurses responsible of general care. This CF includes as a core competence: 'to implement scientific findings for evidence-based practice' ${ }^{6}$. Moreover, the importance of education in EBP is recognized by WHO in the technical guide for Member States, which gives 'European strategic directions for strengthening nursing and midwifery towards Health 2020 goals' ${ }^{7}$.

Nevertheless, the incorporation of EBP into clinical 
nursing has been slow and remains a challenge to the profession. Across different countries, research results have revealed a very restricted level of EBP implementation among nurses ${ }^{8-10}$. There are multiple barriers for the relatively slow paradigm shift to EBP across the globe, including inadequate EBP knowledge and skills, lack of value for and misperceptions about EBP, inadequate EBP resources, administrative support and organizational culture, lack of authority to change patient care procedures, insufficient numbers of EBP mentors within both academic and clinical environments, and negative attitudes toward research as a result of the manner in which students have been taught this content in their academic programs ${ }^{11}$. Also, available evidence indicates that lack of knowledge, confidence in teaching EBP, information and literacy skills, time, resources, frameworks for curricula and support are all barriers experienced by nurse educators ${ }^{12}$. Job satisfaction, values of activism and professionalism, and competencies aimed at the development and professionalization of nursing are also significant factors for EBP beliefs and implementation for employed nurses ${ }^{13,14}$.

There are limited resources to support EBP in European Nursing Curricula. There is no European framework for EBP competency, and no guidelines for EBP teaching. The project 'Providing a Teaching and Learning Open and Innovative Toolkit for Evidence-based Practice to Nursing European Curriculum: (EBP e-Toolkit)' therefore aims to fill this gap in Nursing education across Europe.

The general concept of the EBP e-Toolkit project is to foster and harmonize the teaching and learning of EBP in the European nursing curricula and to produce the acquisition of EBP competence earlier in professional life that can be continued for lifelong self-directed learning also among nurse educators and clinical mentors. Consequently, the nursing students and nurse educators (both academic nursing teachers and clinical mentors supervising students in the clinical placement) are the target groups of this project ${ }^{15}$.

The specific objectives of EBP e-Toolkit project are the following:

1. To analyze the current situation of EBP in European Nursing Curricula.

2. To agree on a European EBP competency framework.

3. To develop guidelines on the recommendations for teaching and learning EBP to the European Nursing Curricula.

4. To develop joint curricula and an EBP e-Toolkit (including an e-course and open educational resources) with a pedagogical strategy for learning and training EBP in nursing oriented to undergraduate nursing students and professional nurses.

5. To validate the innovative educational intervention using EBP e-Toolkit among nursing students and nurse educators (academic and clinical mentors).

\section{METHODS}

The project will be carried out over 3 years and organized along four major outputs, including a dissemination and sustainability plan that will start when the project has been set up. A mixed method research constitutes the main methodological approach that will be applied in the project. This methodology allows researchers to apply the most appropriated methods in the project and depends on target groups (nursing students, educators, clinical mentors) and the specific objectives of each output. This methodology implies the active participation of all research groups, partners, steering committee, nursing students, and educators.

The partnership of this project involves six countries and institutions among countries with specific leadership of the project's individual outputs and activities, as presented in Table 1. The purpose of Output 1 (Slovenia, Czech Republic, Greece, lead partners) is to achieve a general picture on teaching EBP in nursing curricula across Europe and to establish a basis for developing and defining EBP competencies for nurses. The main objective of Output 2 (Italy, lead partner) is to support nursing students and nurses' educators by providing them with materials, resources and means to learn and practice EBP. Output 3 (Poland, lead partner) aims to design the teaching and learning open access online materials for EBP (EBP e-Toolkit) and to develop an innovative EBP education intervention based on the findings of Outputs 1 and 2. Output 4 (Spain, lead partner) aims to test and validate the EBP e-Toolkit as an innovative approach on teaching EBP among partner countries.

All of the partners have substantial experience teaching EBP for a nursing degree and in research on EBP education. The activities have already been agreed upon and distributed among partners. So, responsibilities are divided amongst partners based on their skills and expertise to lead and contribute to the specific activities. Overall, in recognition of the diverse expertise across the partner institutions, the collaborative nature of all aspects of design and delivery is central to the project.

\section{Output 1: Development of guidelines on the recommendations for teaching and learning EBP to Nursing European Curricula}

Output 1 will involve three activities.

\section{Activity 1.1}

For the purposes of Activity 1.1, a common protocol used among partners will be developed in order to collect data regarding teaching and learning EBP in nursing curricula among participating countries. All relevant information will be collected through an e-survey developed for the purposes of the project. Following data gathering and analysis, a report will be developed that will provide information related to teaching EBP in participating countries. This report will describe the past and present situation of EBP teaching in each partner's institution, including mode of delivery of lectures, student activities, and assessment methods. 


\section{Table 1. Outputs and activities of project}

\begin{tabular}{|c|c|c|}
\hline $\begin{array}{l}\text { Institution and } \\
\text { Country }\end{array}$ & Output leadership & Activity leadership \\
\hline $\begin{array}{l}\text { Angela Boškin Faculty } \\
\text { of Health Care, Slovenia }\end{array}$ & $\begin{array}{l}\text { Output } 1 \\
\text { Development of guidelines on the } \\
\text { recommendations for teaching and learning EBP } \\
\text { to European Nursing Curricula. }\end{array}$ & $\begin{array}{l}\text { Activity } 1.1 \\
\text { Report on teaching of EBP in Nursing in partner } \\
\text { institutions and countries. }\end{array}$ \\
\hline $\begin{array}{l}\text { University of Ostrava, } \\
\text { Czech Republic }\end{array}$ & $\begin{array}{l}\text { Output } 1 \\
\text { Development of guidelines on the } \\
\text { recommendations for teaching and learning EBP } \\
\text { to European Nursing Curricula. }\end{array}$ & $\begin{array}{l}\text { Activity } 1.2 \\
\text { Definition of competencies on EBP at different } \\
\text { levels of the nursing role (General Nurse and } \\
\text { Advanced Nurse). }\end{array}$ \\
\hline $\begin{array}{l}\text { Hellenic Mediterranean } \\
\text { University*, Greece }\end{array}$ & $\begin{array}{l}\text { Output } 1 \\
\text { Development of guidelines on the } \\
\text { recommendations for teaching and learning EBP } \\
\text { to European Nursing Curricula. }\end{array}$ & $\begin{array}{l}\text { Activity } 1.3 \\
\text { Systematic review of the methodology for } \\
\text { teaching and learning EBP in nursing. }\end{array}$ \\
\hline UNIMORE, Italy & $\begin{array}{l}\text { Output } 2 \\
\text { Development of an on-line library of re-usable } \\
\text { Open Educational Resources (OER) for EBP. }\end{array}$ & \\
\hline $\begin{array}{l}\text { Medical University of } \\
\text { Warsaw, Poland }\end{array}$ & $\begin{array}{l}\text { Output } 3 \\
\text { Design of the teaching and learning open access } \\
\text { online modules and materials and development of } \\
\text { an innovative EBP education strategy. }\end{array}$ & \\
\hline UMU, Spain & $\begin{array}{l}\text { Output } 4 \\
\text { Development of a web-based e-learning EBP } \\
\text { Toolkit (EBP e-Toolkit): an open access online } \\
\text { resource to improve EBP competence in Nursing. }\end{array}$ & $\begin{array}{l}\text { Activity } 4.1 \\
\text { Development of a web-based e-learning EBP } \\
\text { Toolkit (EBP e-Toolkit) including the OER and the } \\
\text { online materials. } \\
\text { Activity } 4.2 \\
\text { Translation and validation of an instrument to } \\
\text { assess EBP competence among } \\
\text { nursing students and nurses. } \\
\text { Activity } 4.3 \\
\text { Testing and validating the EBP e-ToolKit as an } \\
\text { innovative approach for teaching EBP in the } \\
\text { context of nursing curricula. }\end{array}$ \\
\hline
\end{tabular}

*Former Technological Educational Institute (TEI) of Crete.

\section{Activity 1.2}

For Activity 1.2, a review of literature will be conducted to identify core EBP competencies for nurses. EBP leaders from European countries will develop an initial set of EBP competencies (based on content analysis and review) for nurses at different levels of nursing role in European context. A Delphi survey will be conducted to determine consensus and clarity around the competencies. A Delphi technique will involve using an expert panel that will be asked to complete questions focusing on their opinions and predictions. One round of Delphi will be conducted. The participants will be contacted through e-mail. Each project partner will suggest 4-6 participants for the Delphi technique. International experts will be included that will participate as associated partners. The final set of EBP competencies for nurses in European context will be established.

\section{Activity 1.3}

For Activity 1.3, a systematic review protocol will be developed based on MOOSE guidelines. All partners will be asked to review a set of international databases and also conduct the reviews in their own language. All participants will be asked to summarize the evidence of research and practical experiences, reporting the results and describing the recommendations for teaching EBP. In the end, the recommendations made will form the guidelines for teaching EBP, which will be reviewed by the project's Steering Committee and will be used to inform the development of an open access e-platform.

\section{Output 2: Development of an on-line library of re-usable Open Educational Resources (OER) for EBP}

Partners will discuss and agree on the characteristics, details 
and quality criteria of the resources to be included in an OER for EBP. This will eventually lead to the development of a search protocol with the objectives and methods to undertake a review of the existing online open access EBP resources relevant to all steps of EBP process, namely: a) cultivation of a spirit of inquiry; b) formulation of an answerable question; c) systematic search for research evidence; d) appraisal of the validity, relevance, and applicability of the evidence; e) integration of the research evidence with the clinical expertise of the practitioner and the wishes and desires of the patient/ family; f) implementation of the evidence-based decision and evaluation of the outcomes; and g) dissemination of the results.

The lead partner will gather, organize and appraise the resources made available and develop the on-line library of OER for EBP, which will become available through the project's website platform. A quality appraisal process will then be adopted by users, including nursing students and educators, and members of the project's Steering Committee.

Output 3: Design of the teaching and learning open access online modules and materials and development of an innovative EBP education strategy

Preliminary results from Activities 1.2 and 1.3 of Output 1 will be used to inform the design of online modules and materials for EBP. The partner countries will discuss and agree on the characteristics, format, contents, assignments, evaluation and other details, and quality criteria of the teaching and learning open access online materials. The developed online materials and modules will be translated into all partner languages. Partners will then design an innovative EBP nursing education intervention for nursing students and educators.

Output 4: Development of a web-based e-learning EBP Toolkit, an open access online resource to improve EBP competence in Nursing

Activity 4.1

Activity 4.1 will involve the development of a web-based e-learning EBP Toolkit (EBP e-Toolkit), which will be based on the OER and the online materials develop in Outputs 2 and 3. A Delphi technique will be carried out to get a consensus on the specifications and needs of students and educators to design the web-based EBP e-Toolkit. Three groups will be selected: nursing students, nurse educators, and experts in e-learning. Next, the lead partner will decide on the technical aspects using a web-design expert and will choose a website platform provider with the specifications and needs of the project, and will then design and develop a web-based EBP e-Toolkit that includes the library of re-usable OER and the online materials. A qualitative methodology will be used to assess the feasibility, acceptability and usability of the EBP e-Toolkit and the findings of the pilot study will be used to obtain the final version of the web-based platform.

Activity 4.2

Activity 4.2 includes the translation and validation of 2

Figure 1. Organizational structure of the project

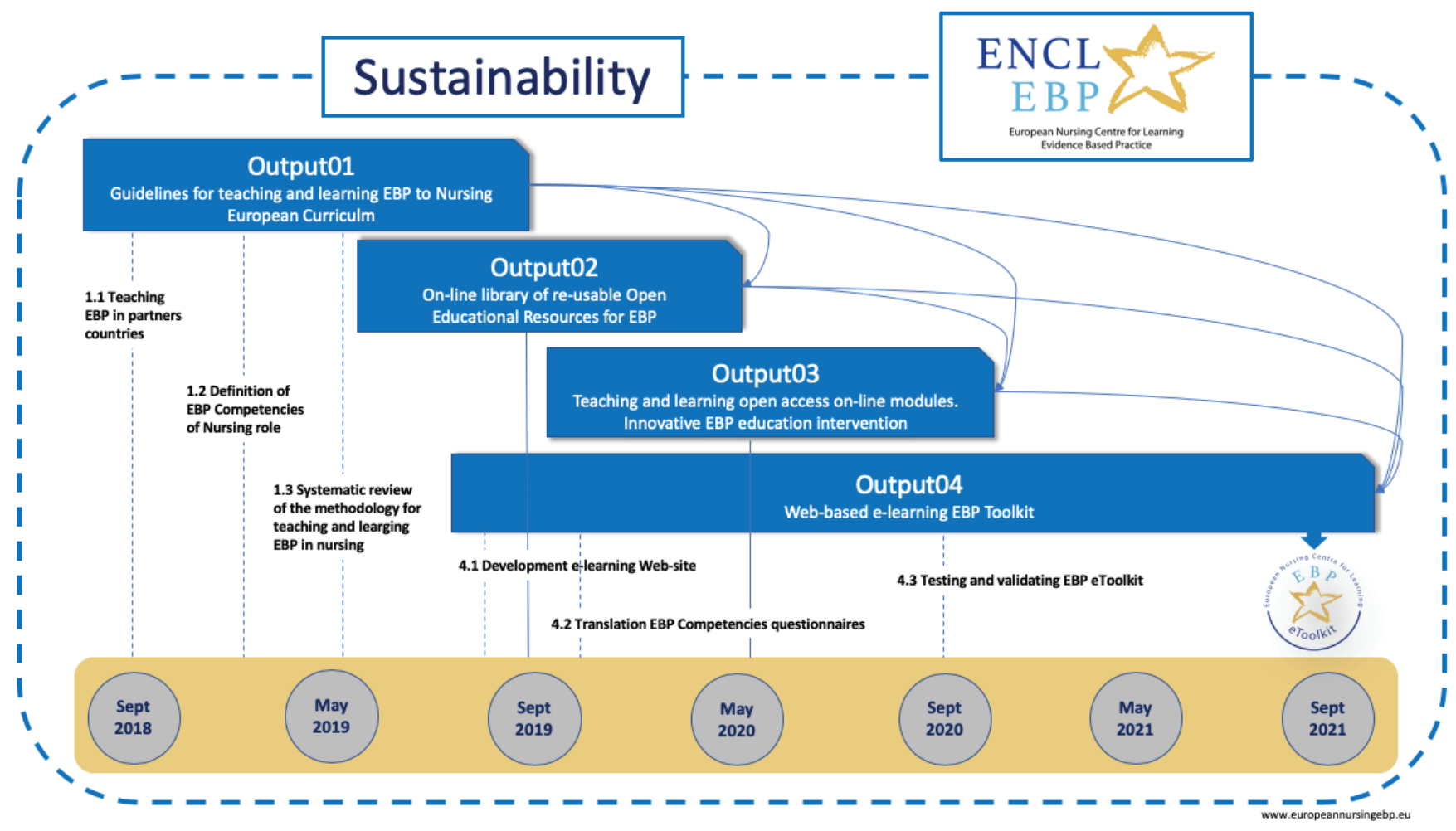


instruments, the Evidence-based Practice Competence Questionnaire for nursing students and nursing professionals (EBP-COQ and EBP-COQ Pro). These questionnaires aim to assess EBP competence among nursing students and nurses and UMU will prepare a protocol for the validation process.

\section{Activity 4.3}

Activity 4.3 involves the validation of the e-learning materials (EBP e-Toolkit) and the innovative approaches on teaching EBP. All nursing students enrolled in the EBP subject where the e-learning materials would be embedded from the partners' countries will be invited to participate (total 1250 nursing students: 220 Spain, 120 Greece, 630 Italy, 80 Czech Republic, 120 Poland, and 80 Slovenia). At the same time, nurses that work as nurse educators and clinical mentors will be sampled and asked to undertake the developed EBP online course, in all the partner countries. It has been agreed that a minimum of 30 nurses in each country will be invited to participate voluntarily (a total of 180 participants). A quasi-experimental design will be carried out before and after, which means that the EBP competence level of all students and nurse educators that take the EBP course will be assessed before and after the course.

The project's outputs, specific activities, timeframe, and the specific interactions between outputs are presented in Figure 1.

\section{DISCUSSION}

The traditional approach to teaching research content in the nursing graduate curricula has focused on educating students to be 'evidence generators' through learning the specific steps of the research process, instead of 'evidence users'16. As a result, students have difficulties in understanding the relevance of research to clinical practice. In the 'evidence user' or EBP approach, students are taught to cultivate a spirit of inquiry by asking clinical questions in a format that will facilitate the search and application of the results of research to get the best answers to their questions and to implement them in the clinical settings. At the same time, clinical mentors' preparation is of vital importance. The mentors should familiarize themselves with the core value of EBP and the process of research utilization. In addition, only when mentors are competent in the concepts and steps of EBP will they be able to incorporate EBP into teaching and support students' EBP learning in clinical settings ${ }^{17,18}$.

The literature on EBP in university nursing education has generally centered on teaching methodologies, mainly describing models developed in North America such as the ARCC-E (advancing research and clinical practice through close collaboration and education ${ }^{16}$, the TRADE EBP model $^{19}$, and the SON (School of Nursing) ladder for success in EBP ${ }^{20}$. However, few studies have evaluated the most effective approach in European settings. EBP teaching to healthcare professionals has mainly been documented in medical students and physicians ${ }^{21-23}$. There is a special need for further research on EBP teaching to undergraduate students of nursing ${ }^{24}$, for whom the optimal approach remains under debate.

Across Europe there are centers dedicated to evidencebased medicine established by universities with an explicit interest in EBP, such as the Centre for Evidence-Based Medicine at the University of Oxford, and the Centre for Reviews and Dissemination at University of York. These centers focus on the promotion of EBP in medical education and practice and therefore have no orientation towards nursing profession improvement and EBP implementation in nursing. Furthermore, the existing medical EBP centers organize training on-site EBP courses, which however focus on medicine and are also offered with high fees that make accessibility very difficult. Such centers are also needed for nurses to support their development of knowledge and awareness of importance of EBP for nursing as a profession.

As recommended by WHO, our project aims to contribute to the promotion and innovation of Europe's Higher Nursing Education by developing guidelines on EBP educational interventions and implementing an evidence-based practice course $^{7}$. The establishment of the European Nursing Centre for Learning Evidence-based Practice (ENCLEBP) will bring together all partner countries to promote and support a framework for teaching and learning EBP competencies in Nursing across Europe. Through the ENCLEBP we will guarantee free access to e-learning materials on EBP and provide a virtual course that can be used in formal and informal learning. The Centre will be open to collaboration with other academic institutions, faculties, professional associations, and other entities interested in teaching and learning EBP in Nursing.

At the completion of the project, we expect to increase the knowledge and skills of EBP and provide the means to develop competency amongst nursing students, nurse educators and clinical mentors to integrate EBP into their teaching and learning. The primary result of the project will be embedding an innovative and online educational strategy on EBP in Nursing curricula across the participating countries using an online toolkit. Also, the EBP e-Toolkit will provide a free and online EBP course oriented to improve the knowledge and skills of nurses' educators, clinical mentors (professional nurses that supervise students in the clinical placements), and registered nurses in general. In addition, the validation of the EBP educational content will provide valuable information on effectiveness, relevance, usability and acceptability of the EBP e-Toolkit developed and implemented, bringing consistency to education in $\mathrm{EBP}^{25}$.

It is expected that the project will impact directly on more than 1250 students in one academic year. All nursing students enrolled in the EBP subject where the e-learning materials would be embedded from the partner countries (220 Spain, 120 Greece, 630 Italy, 80 Czech Republic, 120 Poland and 80 Slovenia) will benefit from this new education intervention. At the same time, we expect that around 180 
nurses that work as nurse educators and clinical mentors will take the developed EBP online course, that means that each year more nurses would be competent to incorporate the EBP in their work as mentors of the nursing students.

At the institutional level, the project will produce changes in the Nursing curricula at 6 institutions from 6 different countries (Faculties and Schools) including the EBP e-Toolkit embedded in the EBP subject as part of the formal syllabus. Also, it is expected that the higher education participants will develop transnational collaborative relationships that will maintain ongoing working partnerships with the establishment of the European Nursing Centre for Learning Evidence-based Practice. At the same time, the project will enhance the quality of caring provided by local and national health systems, as a result of improvement in the competencies of future nurses and clinical mentors in EBP ${ }^{26}$.

The project will provide to local, regional and national stakeholders such as Professional Nurses Associations, Nurse Educators Associations, Professional Unions, Nursing Councils, and Health Care Systems, a European framework for Evidence-based Practice Competency, and guidelines for EBP teaching. A validated EBP e-Toolkit as a virtual platform to teach and learn EBP completely free will become available for use by higher institutions and Health Care Systems and Nurses' Associations. As noted, we provide support to educators, education institutions, health services and clinicians to have the capacity and competence to meet the challenge of providing such EBP education ${ }^{27}$.

The project's outcomes will have an international academic impact, as this will be the first time that six Nursing Centers in Europe test and compare an innovative educational strategy on EBP for undergraduate students and nurse educators. Furthermore, this will be the first time that a tool to measure the competence of EBP, translated and validated in seven languages, and a European Nursing Centre for Learning Evidence-based Practice to support teaching and learning EBP focused on Nursing, will become available for nurses.

\section{CONCLUSIONS}

This project will enhance teaching and learning of EBP in nursing education across Europe, while promoting lifelong learning, both among nursing students and nurse educators. The use of the EBP e-Toolkit will increase nurses' and nursing students' level of EBP competence by providing, for the first time, specific guidelines for EBP teaching.

\section{REFERENCES}

1. Sackett DL. Evidence-based medicine. Semin Perinatol. 1997;21(1):3-5. doi:10.1016/s0146-0005(97)80013-4

2. McGinty J, Anderson G. Predictors of physician compliance with American Heart Association guidelines for acute myocardial infarction. Crit Care Nurs Q. 2008;31(2):161-172. doi:10.1097/01.CNQ.0000314476.64377.12

3. Meijers JMM, Janssen MAP, Cummings GG, Wallin
L, Estabrooks CA, Halfens RYG. Assessing the relationships between contextual factors and research utilization in nursing: systematic literature review. J Adv Nurs. 2006;55(5):622-635. doi:10.1111/j.13652648.2006.03954.x

4. Maljanian R, Caramanica L, Taylor SK, MacRae JB, Beland DK. Evidence-based nursing practice, Part 2: Building skills through research roundtables. J Nurs Adm. 2002;32(2):8590. doi:10.1097/00005110-200202000-00006

5. International Council of Nurses. Evidence based safe nurse staffing. https://www.icn.ch/sites/default/files/inlinefiles/PS_C_\%20Evidence $\% 20$ based $\% 20$ safe $\% 20$ nurse $\% 20$ staffing_1.pdf. Published 2009. Accessed October 8, 2020.

6. European Federation of Nurses Associations. EFN Competency Framework. EFN Guideline to implement Article 31 into national nurses' education programmes. http://www.efnweb.be/wp-content/uploads/EFNCompetency-Framework-19-05-2015.pdf. Published 2015. Accessed October 8, 2020.

7. Jylhä V, Oikarainen A, Perälä ML, Holopainen A. World Health Organization. Facilitating evidence-based practice in nursing and midwifery in the WHO European Region. http://www. euro.who.int/_data/assets/pdf_file/0017/348020/WH06_ EBP_report_complete.pdf?ua=1. Published 2017. Accessed October 8, 2020.

8. Eizenberg MM. Implementation of evidence-based nursing practice: nurses' personal and professional factors?. J Adv Nurs. 2011;67(1):33-42. doi:10.1111/j.13652648.2010.05488.x

9. Boström AM, Rudman A, Ehrenberg A, Gustavsson JP, Wallin L. Factors associated with evidence-based practice among registered nurses in Sweden: a national crosssectional study. BMC Health Serv Res. 2013;13:165. doi:10.1186/1472-6963-13-165

10. Stokke K, Olsen NR, Espehaug B, Nortvedt MW. Evidence based practice beliefs and implementation among nurses: a cross-sectional study. BMC Nurs. 2014;13(1):8. doi:10.1186/1472-6955-13-8

11. Melnyk BM, Fineout-Overholt E. Evidence-Based Practice in Nursing \& Healthcare: A Guide to Best Practice. 4th Edition. Philadelphia: Wolters Kluwer; 2019.

12. Stichler JF, Fields W, Kim SC, Brown CE. Faculty knowledge, attitudes, and perceived barriers to teaching evidence-based nursing. J Prof Nurs. 2011;27(2):92-100. doi:10.1016/j. profnurs.2010.09.012

13. Skela-Savič B, Pesjak K, Lobe B. Evidence-based practice among nurses in Slovenian Hospitals: a national survey. Int Nurs Rev. 2016;63(1):122-131. doi:10.1111/inr.12233

14. Skela-Savič B, Hvalič-Touzery S, Pesjak K. Professional values and competencies as explanatory factors for the use of evidence-based practice in nursing. J Adv Nurs. 2017;73(8):1910-1923. doi:10.1111/jan.13280

15. Ruzafa-Martínez M. EBP e-toolkit project: Providing a teaching and learning open and innovative toolkit for evidence-based practice to nursing european curriculum. 
Central European Journal of Nursing and Midwifery. 2019;10(2):1017-1018. doi:10.15452/cejnm.2019.10.0008

16. Fineout-Overholt E, Melnyk BM, Schultz A. Transforming health care from the inside out: advancing evidence-based practice in the 21st century. J Prof Nurs. 2005;21(6):335344. doi:10.1016/j.profnurs.2005.10.005

17. Zeleníková R, Beach M, Ren D, Wolff E, Sherwood P. Faculty perception of the effectiveness of EBP courses for graduate nursing students. Worldviews Evid Based Nurs. 2014;11(6):401-413. doi:10.1111/wvn.12068

18. Morrison TL, Brennaman L. What do nursing students contribute to clinical practice? The perceptions of working nurses. Appl Nurs Res. 2016;32:30-35. doi:10.1016/j. apnr.2016.03.009

19. Krainovich-Miller B, Haber J, Yost J, Jacobs SK. Evidencebased practice challenge: teaching critical appraisal of systematic reviews and clinical practice guidelines to graduate students. J Nurs Educ. 2009;48(4):186-195. doi:10.3928/01484834-20090401-07

20. Bloom KC, Olinzock BJ, Radjenovic D, Trice LB. Leveling EBP content for undergraduate nursing students. J Prof Nurs. 2013;29(4):217-224. doi:10.1016/j.profnurs.2012.05.015

21. Young T, Rohwer A, Volmink J, Clarke M. What are the effects of teaching evidence-based health care (EBHC)? Overview of systematic reviews. PLoS One. 2014;9(1):e86706. doi:10.1371/journal.pone.0086706

22. Ilic D, Maloney S. Methods of teaching medical trainees evidence-based medicine: a systematic review. Med Educ. 2014;48(2):124-135. doi:10.1111/medu.12288

23. Kyriakoulis K, Patelarou A, Laliotis A, et al. Educational strategies for teaching evidence-based practice to undergraduate health students: systematic review. J Educ Eval Health Prof. 2016;13:34. doi:10.3352/jeehp.2016.13.34

24. Moch SD, Cronje RJ. Part II. Empowering grassroots evidence-based practice: a curricular model to foster undergraduate student-enabled practice change. J Prof Nurs. 2010;26(1):14-22. doi:10.1016/j.profnurs.2009.03.003

25. Horntvedt MET, Nordsteien A, Fermann T, Severinsson E. Strategies for teaching evidence-based practice in nursing education: a thematic literature review. BMC Med Educ. 2018;18(1):172. doi:10.1186/s12909-018-1278-z

26. Bickford CJ. Nursing informatics: Scope and standards of practice. In: Saranto K, et al, eds. Connecting Health and Humans. Studies in Health Technology and Informatics, 146. Amsterdam, Netherlands: IOS Press; 2009. doi:0.3233/978-

\section{ACKNOWLEDGMENTS}

This project is co-funded by the Erasmus+ Programme of the European Union, under grant number 2018-1-ES01-KA203-050216. European Commission support, resulting in the publication of this manuscript, does not constitute an endorsement of its contents, which reflect the views only of the authors. The Commission cannot be held responsible for any use that may be made of the information contained therein.

\section{1-60750-024-7-855}

27. Lehane E, Leahy-Warren P, O'Riordan C, et al. Evidencebased practice education for healthcare professions: an expert view. BMJ Evid Based Med. 2019;24(3):103-108. doi:10.1136/bmjebm-2018-111019 disclosure of potential conflicts of interest. The authors declare that they have no competing interests, financial or otherwise, related to the current work. Dr. Ramos-Morcillo reports grants from Erasmus+ Programme of the European Union, during the conduct of the study.

PROVENANCE AND PEER REVIEW

Not commissioned; externally peer reviewed. 